\title{
EXTREMAL PROPERTIES OF HERMITIAN MATRICES. II
}

\author{
M. MARCUS, B. N. MOYLS, AND R. WESTWICK
}

1. Introduction. Let $H$ be an $n$-square Hermitian matrix with eigenvalues $h_{1} \geqslant h_{2} \geqslant \ldots \geqslant h_{n}$. Fan (2) showed that

$$
\left\{\begin{array}{l}
\max \sum_{j=1}^{k}\left(H x_{j}, x_{j}\right)=\sum_{j=1}^{k} h_{j}, \\
\min \sum_{j=1}^{k}\left(H x_{j}, x_{j}\right)=\sum_{j=1}^{k} h_{n-k+j}
\end{array}\right.
$$

$k=1,2, \ldots, n$, where the max and min are taken over all sets of $k$ orthonormal (o.n.) vectors in unitary $n$-space $V_{n}$. Marcus and McGregor (3) have generalized this result in the case that $H$ is non-negative Hermitian. For vectors $x_{1}, \ldots, x_{r}, r \leqslant n$, in $V_{n}$, let $x_{1} \wedge x_{2} \wedge \ldots \wedge x_{r}$ denote the Grassmann exterior product of the $x_{i}$; it is a vector in $V_{m}$, where

$$
m=\left(\begin{array}{c}
n \\
r
\end{array}\right) \text {. }
$$

The $r$ th compound of $H$ is a Hermitian transformation of $V_{m}$ defined by

$$
C_{r}(H) x_{1} \wedge \ldots \wedge x_{r}=H x_{1} \wedge \ldots \wedge H x_{r} .
$$

For $1 \leqslant r \leqslant k \leqslant n$, denote by $Q_{k r}$ the set of $\left(\begin{array}{c}k \\ r\end{array}\right)$ distinct sequences $w=$ $\left\{i_{1}, \ldots, i_{r}\right\}$ of integers such that $1 \leqslant i_{1}<\ldots<i_{r} \leqslant k$. For a set of vectors $x_{1}, \ldots, x_{k}$ in $V_{n}$, set

$$
x_{w}=x_{i_{1}} \wedge \ldots \wedge x_{i_{r}} .
$$

Let

$$
g=g\left(x_{1}, \ldots, x_{k}\right)=\sum_{w \in Q_{k r}}\left(C_{r}(H) x_{w}, x_{w}\right),
$$

and let $E_{r}\left(a_{1}, \ldots, a_{k}\right)$ be the $r$ th elementary symmetric function of the numbers $a_{1}, \ldots, a_{k}$. Marcus and McGregor showed that

$$
\left\{\begin{array}{l}
\max g=E_{r}\left(h_{1}, \ldots, h_{k}\right) \\
\min g=E_{r}\left(h_{n-k+1}, \ldots, h_{n}\right),
\end{array}\right.
$$

where the max and min are taken over all sets of $k$ o.n. vectors $x_{1}, \ldots, x_{k}$ in $V_{n}$. This result reduces to (1) when $r=1$. In the present note we extend this result to the case where $H$ is an arbitrary Hermitian matrix.

Received July 23, 1958. The work of the first author was supported in part by United States National Science Foundation Research Grant NSF-G 5416; that of the second author by the United States Air Force Office of Scientific Research, Air Research and Development Command; that of the third author by the National Research Council of Canada. 


\section{Results.}

Theorem. Let $1 \leqslant r \leqslant k \leqslant n$ and let $H$ be a Hermitian matrix with eigenvalues $h_{1} \geqslant \ldots \geqslant h_{n}$. Then

$$
\left\{\begin{array}{l}
\max g=\max _{o \leqslant s \leqslant k} E_{r}\left(h_{1}, \ldots, h_{s}, h_{n-k+s+1}, \ldots, h_{n}\right)^{*} \\
\min g=\min _{o \leqslant s \leqslant k} E_{\tau}\left(h_{1}, \ldots, h_{s}, h_{n-k+s+1}, \ldots, h_{n}\right),
\end{array}\right.
$$

where the max and min of $g$ are taken over all sets of $k$ o.n. vectors $x_{1}, \ldots, x_{k}$ in $V_{n}$.

Proof. Let $L=L\left(x_{1}, \ldots, x_{k}\right)$ denote the subspace spanned by the o.n. vectors $x_{1}, \ldots, x_{k}$; and let $P$ be the orthogonal projection of $V_{n}$ into $L$. Then, since $P$ is Hermitian,

$$
\begin{aligned}
g\left(x_{1}, \ldots, x_{k}\right) & =\sum_{w \in Q_{k r}}\left(C_{r}(H) x_{w}, C_{r}(P) x_{w}\right) \\
& =\sum_{w \in Q_{k r}}\left(C_{r}(P H) x_{w}, x_{w}\right) \\
& =\operatorname{trace} \text { of } C_{r}(A) \\
& =E_{r}\left(\lambda_{1}, \ldots, \lambda_{k}\right)
\end{aligned}
$$

where $A$ is the Hermitian transformation $P H$ restricted to $L$, and $\lambda_{1} \geqslant \ldots \geqslant$ $\lambda_{k}$ are the eigenvalues of $A$. It is known $(1, \mathrm{p} .33)$ that for $1 \leqslant j \leqslant k$,

$$
h_{j} \geqslant \lambda_{j} \geqslant h_{n-k+j} .
$$

Let $R_{k}(h)$ be the set of real $k$-tuples $\lambda=\left(\lambda_{1}, \ldots, \lambda_{k}\right), \lambda_{1} \geqslant \ldots \geqslant \lambda_{k}$, satisfying the inequalities (5). Thus the values of $g$ are bounded by the extreme values of $E_{r}(\lambda)=E_{r}\left(\lambda_{1}, \ldots, \lambda_{k}\right)$ as $\lambda$ ranges over $R_{k}(h)$. We shall discuss the maximum value of $E_{r}(\lambda)$ in the following lemmas. Corresponding results hold for the minimum. For the moment we restrict ourselves to the case in which the $h_{j}$ are distinct.

Lemma 1. Let $h_{1}>\ldots>h_{n}$ be given real numbers. Let $1 \leqslant r \leqslant k \leqslant n$, and let

$$
\gamma=\max _{\lambda \in R_{k}(h)} E_{r}(\lambda) .
$$

Then there exists $\mu \in R_{k}(h)$ such that

$$
E_{r}(\mu)=\gamma
$$

and $\mu_{1}>\ldots>\mu_{k}$.

Proof. When $r=1$, the unique solution of (7) is: $\mu_{j}=h_{j}, j=1, \ldots, k$. Hence suppose that $2 \leqslant r \leqslant k$.

Let $T_{k j}(h)$ be the set of $\lambda=\left(\lambda_{1}, \ldots, \lambda_{k}\right) \in R_{k}(h)$ such that $E_{r}(\lambda)=\gamma$ and $\lambda_{1}>\ldots>\lambda_{j}$. Then $T_{k 1}(h)$ is not void by the continuity of the elemen-

*If $s=0$ (or $k$ ) the initial (or terminal) segment is missing. 
tary symmetric functions. Let $m$ be the least integer such that $T_{k m}(h)$ is not void. Then $m$ must equal $k$ for, if not, we shall show that there exists $\nu \in$ $T_{k, m+1}(h)$. Suppose then that $\mu \in T_{k m}(h)$, where

$$
\mu_{1}>\ldots>\mu_{m}=\ldots=\mu_{\mathrm{t}}>\mu_{\mathrm{t}+1} \geqslant \ldots \geqslant \mu_{k} .
$$

From (5) and (8) we have

$$
h_{m}>h_{m+1} \geqslant \mu_{m+1}=\mu_{m}=\mu_{\mathrm{t}-1}=\mu_{\mathrm{t}} \geqslant h_{n-k+\mathrm{t}-1}>h_{n-k+\mathrm{t}} .
$$

Furthermore,

$$
\begin{aligned}
E_{\tau}(\mu) & =\mu_{m} E_{\tau-1}\left(\tilde{\mu}_{m}\right)+E_{\tau}\left(\tilde{\mu}_{m}\right) \\
& =\mu_{\mathrm{t}} E_{\tau-1}\left(\tilde{\mu}_{\mathrm{t}}\right)+E_{\tau}\left(\tilde{\mu}_{\mathrm{t}}\right)
\end{aligned}
$$

where $E_{q}\left(\tilde{\mu}_{\jmath}\right)$ means $E_{q}\left(\mu_{1}, \ldots, \mu_{j-1}, \mu_{j+1}, \ldots, \mu_{k}\right)$. (If $r=k, E_{r}\left(\tilde{\mu}_{j}\right)=0$.) Now $E_{r-1}\left(\tilde{\mu}_{m}\right)=E_{r-1}\left(\tilde{\mu}_{\mathrm{t}}\right)=0$. For, if $E_{r}\left(\tilde{\mu}_{m}\right)>0$, then for $\mu^{\prime}=\left(\mu_{1}, \ldots\right.$, $\left.\mu_{m}+\delta, \ldots, \mu_{k}\right)$,

$$
E_{r}\left(\mu^{\prime}\right)=\left(\mu_{m}+\delta\right) E_{r-1}\left(\tilde{\mu}_{m}\right)+E_{r}\left(\bar{\mu}_{m}\right)>E_{\tau}(\widetilde{\mu})
$$

for $\delta>0$, and, by (8) and (9), $\mu^{\prime} \in R_{k}(h)$ for $\delta$ sufficiently small. This contradicts (6). Similarly, if $E_{r-1}\left(\tilde{\mu}_{\mathrm{t}}\right)<0, E_{\tau}\left(\mu^{\prime \prime}\right)>E_{\tau}(\mu)$ for $\mu^{\prime \prime}=\left(\mu_{1}, \ldots, \mu_{\mathrm{t}}-\right.$ $\left.\delta, \ldots, \mu_{k}\right)$. Hence $E_{r}(\mu)=E_{r}\left(\tilde{\mu}_{m}\right)$ is independent of $\mu_{m}$. Set $\nu_{j}=\mu_{j}$ for $j \neq m$, and choose $\nu_{m}>\mu_{m}$ so that $\nu_{m}<h_{m}$ and $\nu_{m}<\nu_{m-1}$ (if $m>1$ ). Then $\nu \in T_{k, m+1}(h)$.

Lemma 2. Under the hypotheses of Lemma 1,

$$
\gamma=\max _{0<s<k} E_{r}\left(h_{1}, \ldots, h_{s}, h_{n-k+s+1}, \ldots, h_{n}\right) .
$$

.Proof. Since the lemma is obviously true when $r=1$, and also when $k=n$, suppose that $2 \leqslant r \leqslant k<n$. By Lemma $1, T_{k k}(h)$ is not empty. Let $S_{k q}(h)$, $1 \leqslant q \leqslant k$, be the set of those $\lambda \in T_{k k}(h)$ for which $\lambda_{j}=h_{j}, j=1, \ldots, q$; and let $S_{k 0}(h)$ be the set of $\lambda \in T_{k k}(h)$ for which $\lambda_{1}<h_{1}$. Let $s$ be the largest integer such that $S_{k s}(h)$ is not empty. If $s=k$, there is nothing to prove. Otherwise let $\mu \in S_{k s}(h)$. Then

$$
\mu_{j}=h_{n-k+j}, j=s+1, \ldots, k ;
$$

for, if not, we shall show that there exists $\nu \in S_{k, s+1}(h)$, contradicting the choice of $s$.

Let $t$ be the least integer greater than $s$ for which $\mu_{\mathrm{t}}>h_{n-k+\mathrm{t}}$. If $t=s+1$, $h_{\mathrm{t}}>\mu_{\mathrm{t}}$ by the maximality of $s$; while if $t>s+1$

$$
h_{\mathrm{t}} \geqslant h_{n-k+\mathrm{t}-1}=\mu_{\mathrm{t}-1}>\mu_{\mathrm{t}} \text {. }
$$

Thus

$$
h_{\mathrm{t}}>\mu_{\mathrm{t}}>h_{n-k+\mathrm{t}} .
$$

It follows that $E_{r-1}\left(\tilde{\mu}_{t}\right)=0$, since otherwise we could vary $\mu_{t}$ up or down to increase $E_{\tau}(\mu)$ (see (10)) while keeping $\mu$ in $T_{k k}(h)$. 
Thus

$$
E_{r}(\mu)=E_{r}\left(\tilde{\mu}_{t}\right)
$$

Set

$$
\begin{aligned}
\nu_{j} & =\mu_{j}, j=1, \ldots, s, \\
\nu_{s+1} & =h_{s+1}, \\
\nu_{j} & =\mu_{j-1}, j=s+2, \ldots, t, \\
\nu_{j} & =\mu_{j}, j=t+1, \ldots, k,
\end{aligned}
$$

In effect, $\mu_{\mathrm{t}}$ is replaced by $h_{s+1}$, and the resulting $\mu_{j}$ 's are re-indexed to restore the ordering. By $(12), E_{r}(\nu)=E_{r}(\mu)$. It is then a straightforward matter to verify that $\nu \in S_{k, s+1}(h)$. This completes the proof of the lemma.

We are now in a position to complete the proof of the theorem. If the eigenvalues of $H$ are distinct, then for o.n. $x_{1}, \ldots, x_{k}$,

$$
\begin{aligned}
g\left(x_{1}, \ldots, x_{k}\right) & \leqslant \max _{\lambda \in R_{k}(h)} E_{r}(\lambda) \\
& =E_{r}\left(h_{1}, \ldots, h_{s}, h_{n-k+s+1}, \ldots, h_{n}\right) .
\end{aligned}
$$

for some $s, 0 \leqslant s \leqslant k$. Now $g$ attains this value for o.n. eigenvectors $y_{1}, \ldots, y_{k}$ corresponding to $h_{1}, \ldots, h_{s}, h_{n-k+s+1}, \ldots, h_{n}$, respectively. Thus

$$
\max g=\max _{0 \leqslant s \leqslant k} E_{r}\left(h_{1}, \ldots, h_{s}, h_{n-k+s+1}, \ldots, h_{n}\right) .
$$

A similar result holds for the minimum. That these results remain valid when the eigenvalues of $H$ are not all different follows by a continuity argument.

\section{REFERENCES}

1. R. Courant and D. Hilbert, Methods of mathematical physics, vol. 1 (New York, 1953).

2. Ky Fan, On a theorem of Weyl concerning eigenvalues of linear transformations, $I$, Proc. N.A.S. (U.S.A.), 35 (1949), 652-5.

3. M. Marcus and J. L. McGregor, Extremal properties of Hermitian matrices, Can. J. Math., 8 (1956), 524-31.

The University of British Columbia 